\title{
Molecular Signaling Involved in Entry and Exit of Malaria Parasites from Host Erythrocytes
}

\author{
Shailja Singh ${ }^{1,2}$ and Chetan E. Chitnis ${ }^{1,3}$ \\ ${ }^{1}$ Department of Parasites and Insect Vectors, Institut Pasteur, 75015 Paris, France \\ ${ }^{2}$ Shiv Nadar University, Gautam Buddha Nagar, Uttar Pradesh 201314, India \\ ${ }^{3}$ Malaria Group, International Centre for Genetic Engineering and Biotechnology (ICGEB), New Delhi \\ 110067, India \\ Correspondence: cchitnis@gmail.com
}

During the blood stage, Plasmodium spp. merozoites invade host red blood cells (RBCs), multiply, exit, and reinvade uninfected RBCs in a continuing cycle that is responsible for all the clinical symptoms associated with malaria. Entry into (invasion) and exit from (egress) RBCs are highly regulated processes that are mediated by an array of parasite proteins with specific functional roles. Many of these parasite proteins are stored in specialized apical secretory vesicles, and their timely release is critical for successful invasion and egress. For example, the discharge of parasite protein ligands to the apical surface of merozoites is required for interaction with host receptors to mediate invasion, and the timely discharge of proteases and pore-forming proteins helps in permeabilization and dismantling of limiting membranes during egress. This review focuses on our understanding of the signaling mechanisms that regulate apical organelle secretion during host cell invasion and egress by malaria parasites. The review also explores how understanding key signaling mechanisms in the parasite can open opportunities to develop novel strategies to target Plasmodium parasites and eliminate malaria.

$P_{c}^{l}$ asmodium falciparum has a complex life cycle involving multiple stages in two hosts, namely, the vertebrate human host and the invertebrate Anopheles mosquito, which also serves as the vector for transmission. P. falciparum sporozoites are introduced into the human host following a bite by an infected female Anopheles mosquito in search of a blood meal. The injected sporozoites traverse through the bloodstream to the liver where they infect hepatocytes, multiply, and differentiate into merozoites. The merozoites are released into the bloodstream in membrane-bound packets called merosomes, which protect the parasite from host immune mechanisms. Merozoites emerge from merosomes in the bloodstream and go on to invade host red blood cells (RBCs) within which they develop and multiply by schizogeny over a period of $36-48$ hours. Once the next generation of merozoites has developed, the mature schizont ruptures and merozoites egress in a highly synchronized manner. Merozoite egress requires disruption of the RBC cytoskeleton and rupture of multi-

Editors: Dyann F. Wirth and Pedro L. Alonso

Additional Perspectives on Malaria: Biology in the Era of Eradication available at www.perspectivesinmedicine.org

Copyright (C) 2017 Cold Spring Harbor Laboratory Press; all rights reserved; doi: 10.1101/cshperspect.a026815

Cite this article as Cold Spring Harb Perspect Med 2017;7:a026815 
ple limiting membranes (Salmon et al. 2001; Wickham et al. 2003). Key parasite effectors that mediate egress include proteases as well as perforin-like proteins (Blackman 2008; Roiko and Carruthers 2009, 2013; Agarwal et al. 2012; Garg et al. 2013). Many of these effectors are localized in apical organelles and are secreted in a timely, regulated manner to initiate egress of merozoites from mature schizonts. The released merozoites go on to invade uninfected erythrocytes to continue blood stage multiplication.

Invasion of host erythrocytes by $P$. falciparum merozoites is also a complex multistep process that involves initial attachment, apical reorientation, junction formation, development of a nascent vacuole, and movement of the parasite into the vacuole, followed by resealing of the vacuole so that the parasite is surrounded by a vacuolar membrane at the end of invasion (Dvorak et al. 1975; Aikawa et al. 1978; Cowman and Crabb 2006; Gilson and Crabb 2009; Riglar et al. 2011; Baum 2013). Many of the parasite ligands that bind host receptors to mediate these steps during invasion are initially localized in the micronemes and rhoptries at the apical end of merozoites. They are secreted to the surface of the merozoite in a highly regulated manner to enable receptor binding and invasion (Singh et al. 2010; Gaur and Chitnis 2011; Riglar et al. 2011; Singh and Chitnis 2012; Sharma and Chitnis 2013).

Here, we will review our current understanding of the signals and signaling mechanisms that regulate the highly coordinated processes of parasite egress and host cell invasion. This review will focus on our understanding of the key signaling mechanisms that trigger the release of parasite proteins from apical organelles to mediate parasite egress and invasion. We will include information from both malaria parasites as well as the related apicomplexan parasite, Toxoplasma gondii, which shares many common features in the process of host cell invasion. Finally, we will discuss how a better understanding of signaling processes during invasion and egress may provide novel targets for intervention to interrupt the malaria parasite life cycle and eliminate malaria.

\section{MOLECULAR PLAYERS AND SIGNALS FOR EGRESS OF Plasmodium falciparum MEROZOITES FROM MATURE SCHIZONTS}

Egress of $P$. falciparum merozoites from RBCs requires breaching of multiple barriers including the parasitophorous vacuole membrane (PVM), the host cytoskeleton, and the host plasma membrane (HPM). Parasite egress is synchronized with completion of its replicative cycle when next generation of invasive parasites have developed fully. Several parasite proteases have been identified as key effectors of the egress process in apicomplexan blood-stage parasites (Blackman 2008; Roiko and Carruthers 2009). In addition, perforin-like proteins with homology with mammalian perforins are involved in egress by T. gondii tachyzoites (TgPLP) as well as P. falciparum merozoites (PfPLP) (Kafsack et al. 2009, 2010; Garg et al. 2013). The PLPs and the subtilisin-like protease, PfSub1, which mediate egress, are located in micronemes and exonemes, respectively (Yeoh et al. 2007; Agarwal et al. 2012; Garg et al. 2013). What are the signals and signaling mechanisms that trigger release of these effectors to initiate egress?

The phytohormone abscisic acid (ABA) was identified as the internal signal that triggers egress of $T$. gondii tachyzoites (Nagamune et al. 2008). In plants, ABA acts as a hormone that mediates growth and responses to environmental cues through cyclic ADP-ribose (cADPR) and calcium $\left(\mathrm{Ca}^{2+}\right)$ fluxes (Wu et al. 1997). In T. gondii, ABA induces the production of cADPR, which activates $\mathrm{Ca}^{2+}$ release from an internal membrane-bound pool (probably the endoplasmic reticulum [ER]) leading to secretion of microneme proteins such as TgPLP (Chini et al. 2005; Nagamune et al. 2008). Although the parasite genome has candidate genes for ABA synthesis, the presence of the entire biosynthetic pathway remains to be confirmed. Reflecting its plant heritage, ABA is most likely synthesized in the apicoplast, an organelle found in apicomplexans that is derived from an algal endosymbiont. Interestingly, ABA levels spike late in schizogeny just before parasite egress. Thus, the steep increase in production of $\mathrm{ABA}$ at the end of the replicative cycle might 
serve as an intrinsic cue for egress. The herbicide fluridone, which inhibits ABA synthesis, blocks egress of T. gondii tachyzoites (Nagamune et al. 2008). Furthermore, mice treated with fluridone survive inoculation with a lethal dose of Toxoplasma. Fluridone also blocks $\mathrm{Ca}^{2+}$-mediated egress of $P$. falciparum merozoites from mature schizonts (S Singh and CE Chitnis, unpubl.), suggesting a conserved role for ABA in regulating egress in apicomplexan parasites (Fig. 1).

Rupture of limiting membranes during egress exposes $T$. gondii tachyzoites to a drop in the concentration of $\mathrm{K}^{+}\left(\left[\mathrm{K}^{+}\right]\right)$, which activates tachyzoite motility leading to rapid exit of mature tachyzoites (Moudy et al. 2001). Precisely how the tachyzoite senses $\left[\mathrm{K}^{+}\right]$is not known, although phospholipase C (PLC), cytosolic $\left[\mathrm{Ca}^{2+}\right]$ levels, and several $\mathrm{Ca}^{+2}$-responsive proteins are thought to play a role (Moudy et al. 2001). The levels of $\left[\mathrm{K}^{+}\right]$in the environment may be a natural external cue for tachyzoite egress at the end of the replicative cycle. However, parasites have also been observed to exit host cells in the absence of parasite motility or low $\left[\mathrm{K}^{+}\right]$(Lavine and Arrizabalaga 2008; Abkarian et al. 2011). In case of malaria parasites, osmotic stress and host cell membrane tension may induce egress (Abkarian et al. 2011). Under normal circumstances, however, it is likely that the parasite uses intrinsic as well as extrinsic cues such as $\mathrm{ABA}$ and $\left[\mathrm{K}^{+}\right]$, respectively, as signals to trigger escape.

Several recent studies have provided compelling evidence for a role for proteases in egress of Plasmodium spp. merozoites (Blackman 2004, 2008). Protease inhibitors have proved invaluable tools in analyzing the role of proteases in Plasmodium biology and have shown that egress is a protease-dependent function. Although multiple models for merozoite egress have been proposed, evidence is accumulating in support of a model in which the PVM is disrupted before the RBC membrane (RBCM) (Winograd et al. 1999; Wickham et al. 2003). Further evidence for a model in which the parasite egresses in a stepwise manner came from observations that disruption of each limiting membrane is sensitive to different protease in- hibitors (Salmon et al. 2001; Wickham et al. 2003; Soni et al. 2005; Millholland et al. 2011). Whereas the cysteine and serine protease inhibitors, leupeptin and antipain, block RBCM disruption, the cysteine protease inhibitor E-64 blocks PVM disruption (Glushakova et al. 2008). The targets of these inhibitors, however, remain to be determined.

Other proteases involved in egress include DPAP3, a cathepsin-C like cysteine protease, which is required for maturation of PfSUB1, a subtilisin-like protease implicated in egress (Arastu-Kapur et al. 2008). PfSUB1 is an essential protein that is expressed in late-blood-stage parasites and is secreted into the PV from apical merozoite organelles called exonemes before egress (Yeoh et al. 2007). Once in the PV, PfSUB1 processes serine-rich antigen 5 (SERA5) and potentially other serine-repeat antigen (SERA) proteins (Yeoh et al. 2007; Arastu-Kapur et al. 2008). The SERAs are PV proteins with papainlike putative protease domains. Although evidence of proteolytic activity has yet to be shown by SERAs, it is possible that PfSub1 processing of SERA5 and other SERAs results in their activation and proteolysis of host cytoskeletal proteins to aid in parasite egress (Yeoh et al. 2007). Alternatively, SERAs might activate other parasite or host proteins that promote parasite egress. PfSERA5 processing from a precursor to mature form is linked to egress because this putative activation step occurs coincident with parasite exit from the erythrocyte (Blackman 2008). Inhibition of PfSERA5 by a cyclical peptide halts late-stage development of intraerythrocytic parasites possibly by blocking egress (Fairlie et al. 2008).

A host calcium-dependent protease, calpain-1, is also required for efficient parasite egress of Plasmodium spp. merozoites and Toxoplasma tachyzoites (Olaya and Wasserman 1991; Chandramohanadas et al. 2009). Treatment of $P$. falciparum infected erythrocytes with an irreversible cysteine protease inhibitor, DCG04, did not affect parasite maturation but prevented parasite release from the host cell (Chandramohanadas et al. 2009). Selective extraction of treated cells identified host calpain- 1 as the target of the inhibitor. Calpain-1 was observed in 

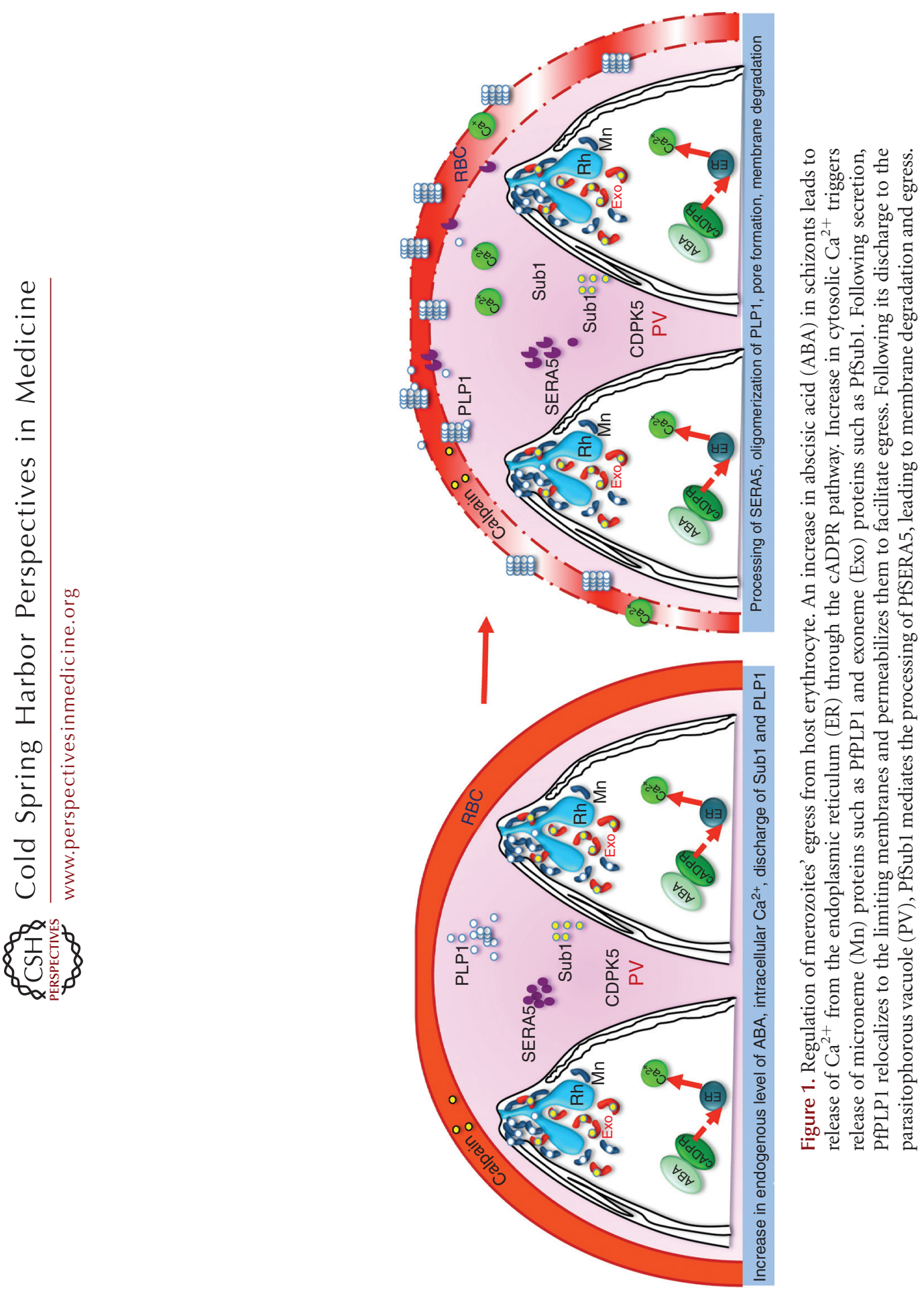
the cytoplasm of the infected host cell until the schizont stage of parasite growth, when it relocated to the membrane following binding to $\mathrm{Ca}^{2+}$ and activation. Calpain-1 depletion from erythrocytes prevented parasite egress and led to growth arrest in the schizont stage, whereas reconstitution with recombinant calpain-1 restored normal growth development (Chandramohanadas et al. 2009). Human host cells treated with siRNA for host calpains (calpains 1 and 2) showed that it was required for T. gondii egress (Chandramohanadas et al. 2009).

\section{ROLE OF APICAL ORGANELLE DISCHARGE IN REGULATION OF PARASITE EGRESS}

Until recently, micronemes, the tubular shaped secretory organelles at the apical end of apicomplexan parasites, were thought to primarily contain adhesins that bind to cognate receptors on host cells during invasion. However, recently it has been shown that they also contain proteins that may be involved in parasite egress such as the pore-forming T. gondii perforin-like protein (TgPLP1) (Kafsack et al. 2009, 2010). It has been known for some time that microneme secretion is triggered by elevation of cytosolic $\mathrm{Ca}^{2+}$. More recently, $\mathrm{Ca}^{2+}$ has also been implicated in regulating microneme secretion, which is required for parasite egress (Kafsack et al. 2009; Garg et al. 2013). Some of the molecular players in the calcium signaling pathway that play key roles in microneme secretion include TgCDPK1 and $\operatorname{TgCDPK} 3$, members of the calcium-dependent protein kinase family (Lourido et al. 2010). In a parallel study, using whole genome sequencing of Toxoplasma egress mutants, TgCDPK3 was found to be an essential factor for $\mathrm{Ca}^{2+}$-induced egress from host cells. Because TgCDPK3 localizes to the periphery of the parasite, $\operatorname{TgCDPK} 3$ is proposed to be part of a signaling pathway that senses changes in the environment leading to egress (Lourido et al. 2012; McCoy et al. 2012). In case of $P$. falciparum, PfCDPK1 has been shown to play a role in microneme secretion (Bansal et al. 2013). In addition, although conditional deletion of PfCDPK5 results in a block in P. falciparum merozoite egress from mature schizonts, loss of PfCDPK5 does not impair RBC invasion (Dvorin et al. 2010). Whether PfCDPK5 is responsible for regulating release of an apical organelle that is required for egress remains to be determined.

The activation of cGMP-dependent protein kinase $\mathrm{G}$ (TgPKG) has also been shown to induce microneme secretion and egress. Compound 1, a selective apicomplexan PKG inhibitor, was used to establish the role of TgPKG in microneme secretion and egress (Wiersma et al. 2004). Treatment of $P$. falciparum schizonts with compound 1 results in a block in schizont rupture, which does not occur when transgenic parasites with a gatekeeper mutation are treated with compound 1 (Taylor et al. 2010; Collins et al. 2013). Phosphoproteomic analysis of merozoites with and without treatment with Compound 1 suggests that a cGMP-dependent kinase serves as a signaling hub that regulates key processes in invasion and egress of $P$. falciparum merozoites (Alam et al. 2015). Interestingly, in contrast to PfCDPK5 knockdown parasites, Compound 1-treated merozoites were not invasive (Dvorin et al. 2010). Collectively, these findings describe the complex signaling pathways that regulate egress and suggest that the different pathways cross talk with each other enabling the parasite to respond to multiple signals to orchestrate a timely escape from host cells.

\section{MOLECULAR PLAYERS FOR ERYTHROCYTE INVASION BY Plasmodium spp. MEROZOITES}

Following egress and release from mature schizonts, Plasmodium merozoites go on to invade uninfected erythrocytes to initiate another round of intracellular replication. Erythrocyte invasion by Plasmodium spp. merozoites has been studied extensively by live cell imaging and electron microscopy (Dvorak et al. 1975; Aikawa et al. 1978; Riglar et al. 2011; Hanssen et al. 2013). After attachment to erythrocytes, merozoites reorient and develop a tight junction between their apical ends and target erythrocytes (Aikawa et al. 1978; Besteiro et al. 2011; Riglar et al 2011). These steps are mediated by multiple interactions between parasite 
protein ligands and host receptors (Gaur and Chitnis 2011; Cowman et al. 2012). The identity of host receptors involved in erythrocyte invasion came initially from studies that used genetically deficient erythrocytes or enzyme-treated erythrocytes for invasion studies. The first report on essentiality of a molecular interaction between Plasmodium spp. merozoites and erythrocytes during invasion was based on the observation that Duffy negativity in West Africa was associated with the absence of $P$. vivax infection (Miller et al. 1976). It was shown that P. vivax is dependent on interaction with the Duffy blood group antigen (also known as Duffy antigen receptor for chemokines [DARC]) to establish a blood-stage infection in humans (Miller et al. 1976; Horuk et al. 1993). However, recent studies have identified $P$. vivax strains in Kenya, Brazil, and Madagascar that can infect Duffy-negative individuals suggesting that $P$. vivax may invade using alternative invasion pathways (Ryan et al. 2006; Cavasini et al. 2007; Ménard et al. 2010). In case of $P$. falciparum, glycophorins A, B, C (GPA, GPB, GPC) were identified as receptors for invasion (Gaur and Chitnis 2011). However, no human erythrocyte lacking an individual receptor has been found to be completely refractory to invasion by $P$. falciparum indicating redundancy in invasion pathways. Following identification of host receptors, a family of erythrocyte-binding proteins (EBPs) that includes $P$. vivax and $P$. knowlesi Duffybinding proteins ( $\mathrm{PvDBP}$ and $\mathrm{PkDBP})$ and $P$. falciparum erythrocyte-binding antigens, EBA175, EBA-140, EBA-181, and EBL-1, were identified (Gaur and Chitnis 2011). GPA, GPB, and GPC have been shown to bind EBA-175, EBA140, and EBL-1, respectively (Gaur and Chitnis 2011).

Another $P$. falciparum multigene family, which shares homology with $P$. vivax reticulocyte-binding proteins and binds erythrocytes, is the PfRH family of $P$. falciparum proteins (Gaur and Chitnis 2011). The PfRH protein family includes PfRH1a, PfRH1b PfRH2a, PfRH2b, PfRH4, and PfRH5. Genetic knockout studies have confirmed that PfRH2a/b and PfRH4 mediate sialic acid independent invasion pathways (Cowman et al. 2012). Complement receptor 1
(CR1) has recently been shown to serve as the erythrocyte receptor for PfRH4 (Tham et al. 2010). PfRH5 has also been shown to mediate invasion of Aotus erythrocytes by $P$. falciparum (Hayton et al. 2008). Basigin has been recently shown to be a receptor for PfRH5 binding (Crosnier et al. 2011). Other merozoite proteins implicated in invasion include thrombospondin-related anonymous protein (TRAP) homologs such as MTRAP and PTRAMP that contain the characteristic thrombospondin repeat (TSR) adhesive domains (Bartholdson et al. 2012; Siddiqui et al. 2013). Recent work has shown that the conserved apical membrane antigen-1 (PfAMA1) in complex with the rhoptry neck proteins (PfRON2 and PfRON4) localizes to the junction (Richard et al. 2010; Besteiro et al. 2011; Srinivasan et al. 2011). The exported PfRON2 protein is inserted into the erythrocyte membrane and interacts with secreted PfAMA1 during invasion. Other invasion-related proteins include two protein complexes, the highmolecular-weight (HMW) complex composed of RhopH1, RhopH2, and RhopH3 and the low-molecular-weight (LMW) complex composed of RAP1, RAP2, and RAP3. Apart from these adhesins and their interacting parasite ligands, Plasmodium spp. merozoites have a conserved molecular machinery for motility, which comprises of a central actin-myosin motor located in the pellicle of the parasite that is linked with both a surface adhesin and the inner membrane complex (IMC) and is likely to drive the invasion process (Sharma and Chitnis 2013). Recent work has shown that PfRON2-PfAMA1 interaction may not be essential for junction formation and there may be alternative pathways for invasion that are not dependent on the actin-myosin motor (Bargieri et al. 2014).

\section{MOLECULAR SIGNALS THAT REGULATE APICAL ORGANELLE DISCHARGE DURING INVASION}

As discussed above, many of the parasite proteins that bind erythrocyte receptors to mediate different steps in the invasion process are localized to the micronemes and rhoptries and must be translocated to the merozoite surface in a 
regulated manner to enable receptor engagement and invasion. For example, PfAMA1 and PfEBA175 are localized in the micronemes, whereas PfRON2, and PfRON4 are localized in the rhoptry neck and PfRH2, PfRH5, PfRAP1-3, and PfRhop1-3 are localized in the rhoptry bulb. The timely discharge of miconeme and rhoptry proteins is essential for successful invasion by $P$. falciparum merozoites (Singh et al. 2010; Riglar et al. 2011; Hanssen et al. 2013).

What are the external signals and internal signaling pathways that mediate discharge of microneme and rhoptry proteins in Plasmodium merozoites and Toxoplasma tachyzoites during invasion? Studies with T. gondii tachyzoites first revealed that the levels of free cytosolic $\mathrm{Ca}^{2+}$ are high in tachyzoites during the process of gliding and are restored to basal levels following attachment to target cells during invasion (Carruthers and Sibley 1999; Carruthers et al. 1999; Lovett et al. 2002; Moreno and Docampo 2003). Similarly, cytosolic $\mathrm{Ca}^{2+}$ levels are high in $P$. falciparum merozoites and are restored to basal levels following attachment to target erythrocytes during invasion indicating that $\mathrm{Ca}^{2+}$ may regulate processes such as vesicular secretion during invasion (Singh et al. 2010). Indeed, treatment of $P$. falciparum merozoites with A23187 triggers an increase in cytosolic $\mathrm{Ca}^{2+}$ and secretion of microneme proteins such as PfAMA1 and PfEBA-175 but has no effect on secretion of rhoptry proteins (Singh et al. 2010). Importantly, the $\mathrm{Ca}^{2+}$ ionophore, A23187, induces microneme secretion in absence of extracellular $\mathrm{Ca}^{2+}$, which indicates that $\mathrm{Ca}^{2+}$ from intracellular stores is sufficient for secretion of microneme proteins (Singh et al. 2010). Ethanol and other short-chain alcohols elevate cytosolic $\mathrm{Ca}^{2+}$ in T. gondii tachyzoites to stimulate microneme secretion (Carruthers and Sibley 1999; Carruthers et al. 1999). Treatment of tachyzoites with thapsigargin, which inhibits sarcoplasmic/ endoplasmic reticulum calcium ATPase (SERCA) and blocks pumping of $\mathrm{Ca}^{2+}$ from the cytosol back to the endoplasmic reticulum (ER), increases cytosolic $\left[\mathrm{Ca}^{2+}\right]$ levels and triggers secretion of microneme proteins. In contrast, chelation of cytosolic $\mathrm{Ca}^{2+}$ by treatment of
T. gondii tachyzoites or $P$. falciparum merozoites with BAPTA-AM inhibits microneme release and invasion of host cells (Singh et al. 1990; Carruthers and Sibley 1999; Carruthers et al. 1999). $\mathrm{Ca}^{2+}$ is stored in organelles such as the ER, mitochondria, and acidocalcisomes in apicomplexan parasites (Garcia 1999; Alleva and Kirk 2001). Treatment of $T$. gondii tachyzoites with ethanol raises levels of intracellular inositol triphosphate $\left(\mathrm{IP}_{3}\right)$, which trigger $\mathrm{Ca}^{2+}$ release by binding to $\mathrm{IP}_{3}$-receptor gated $\mathrm{Ca}^{2+}$ channels $\left(\mathrm{IP}_{3} \mathrm{R}\right)$ on the ER. Furthermore, xestospongin C, an $\mathrm{IP}_{3} \mathrm{R}$ antagonist, inhibits ethanol-triggered release of $\mathrm{Ca}^{2+}$ and microneme secretion in T. gondii suggesting the presence of $\mathrm{IP}_{3} \mathrm{R}$ (Lovett et al. 2002). The presence of PLC has been shown in T. gondii and PLC transcripts have been detected in P. falciparum blood-stage parasites. Moreover, an inhibitor of PLC has been shown to block $\mathrm{Ca}^{2+}$ release in T. gondii and $P$. falciparum providing further evidence for the presence of an $\mathrm{IP}_{3}$-mediated pathway for $\mathrm{Ca}^{2+}$ release in these parasites (Carruthers and Sibley 1999; Carruthers et al. 1999; Lovett et al. 2002; Moreno et al. 2003; Vaid et al. 2008; Singh et al. 2010). Treatment of T. gondii with ryanodine or caffeine, which are agonists of the ryanodine receptor (RyR)-gated $\mathrm{Ca}^{2+}$ channel on ER, also leads to an increase in cytosolic $\mathrm{Ca}^{2+}$ and microneme secretion (Lovett et al. 2002). The ER thus appears to be the primary intracellular store from which $\mathrm{Ca}^{2+}$ is mobilized. Endogenous cyclic ADP ribose (cADPR), which can bind RyR to induce calcium release, was detected in T. gondii extracts suggesting that it may also play a role as a second messenger to regulate calcium levels and microneme secretion (Chini et al. 2005). Moreover, treatment of $T$. gondii tachyzoites with RyR antagonists, 8-bromo-cADPR and ruthenium red, blocks microneme secretion (Chini et al. 2005). Although use of modulators has provided pharmacological evidence for the presence of both $\mathrm{IP}_{3} \mathrm{R}$ and RyR in both $P$. falciparum and T. gondii, the genes encoding $\mathrm{IP}_{3} \mathrm{R}$ and $\mathrm{RyR}$ have not yet been identified.

The increase in cytosolic $\left[\mathrm{Ca}^{2+}\right]$ levels activates $P$. falciparum calcium-dependent protein kinase 1 (PfCDPK1) and T. gondii CDPK1 
(TgCDPK1), which play central roles in orchestrating many of the processes required for invasion of host cells, including microneme release and the activation of the actin-myosin motor (Green et al. 2008; Kato et al. 2008b; Lourido et al. 2010; Bansal et al. 2013). Recently, a screen for molecules required for invasion of host cells by $T$. gondii identified a DOC2 protein that is required for microneme secretion, presumably by mediating $\mathrm{Ca}^{2+}$-dependent vesicular docking activity (Farrell et al. 2012). The P. falciparum ortholog of this protein is also required for the invasion of red blood cells and release of micronemes (Farrell et al. 2012). Independent of $\mathrm{Ca}^{2+}$ release, chemical genetic and phosphoproteomics evidence suggests that cyclic guanosine monophosphate (cGMP) and the cGMPdependent kinase, $\mathrm{PKG}$, also play an essential role in microneme release and invasion (Moon et al. 2009; Alam et al. 2015).

What might be the external signals that trigger secretion of microneme and rhoptry proteins during invasion? We have shown that exposure of $P$. falciparum merozoites to a low- $\left[\mathrm{K}^{+}\right]$ environment as found in blood plasma can serve as a natural signal that triggers an increase in cytosolic $\left[\mathrm{Ca}^{2+}\right]$ through the PLC pathway leading to secretion of microneme proteins such as PfEBA175 and PfAMA1 to the merozoite surface (Singh et al. 2010). Following translocation to the merozoite surface, binding of EBA175 to GPA restores basal $\left[\mathrm{Ca}^{2+}\right]$ levels and triggers release of rhoptry proteins such as PfRH2b, Clag3.1, and PfTRAMP (Singh et al. 2010; Siddiqui et al. 2013). Secretion of microneme and rhoptry proteins to the merozoite surface is thus a two-step process as shown in Figure 2. Microneme proteins have also been shown to play a role in rhoptry secretion in T. gondii. Deletion of microneme protein MIC8 resulted in a block in rhoptry secretion and parasite growth (Kessler et al. 2008). The cytoplasmic domain of microneme proteins was implicated in playing a role to trigger rhoptry secretion (Kessler et al. 2008).

The pathway by which a low- $\left[\mathrm{K}^{+}\right]$environment triggers an increase in cytosolic $\left[\mathrm{Ca}^{2+}\right]$ in P. falciparum merozoites is regulated by another ubiquitous second messenger, namely, $3^{\prime}-5^{\prime} \mathrm{cy}-$ clic adenosine monophosphate (cAMP) (Dawn et al. 2014). The transfer of $P$. falciparum merozoites to a low- $\left[\mathrm{K}^{+}\right]$medium activates the parasite carbonic anhydrase (PfCA), which produces $\mathrm{H}^{+}$ions and bicarbonate $\left(\mathrm{HCO}_{3}^{-}\right)$to balance cytosolic pH (Dawn et al. 2014). Increase in cytosolic bicarbonate levels activates bicarbonate-sensitive soluble adenylate cyclase, PfAC $\beta$ leading to an increase in cAMP levels, which activates protein kinase A (PfPKA) as well as the cAMP responsive GTP exchange protein called exchange protein activated by cAMP (PfEPAC) (Fig. 2) (Dawn et al. 2014). In other eukaryotic cells, activated EPAC transfers GTP to Rap1 GTPase, which then activates PLC (Gloerich and Bos 2010). Use of specific agonists as well as inhibitors has shown that the EPAC pathway for activation of PLC following an increase in cAMP is present and is responsible for the increase in $\left[\mathrm{Ca}^{2+}\right]$ when merozoites are exposed to a low- $\left[\mathrm{K}^{+}\right]$environment following egress from schizonts. In addition to regulating levels of free $\left[\mathrm{Ca}^{2+}\right]$ in merozoites, cAMP also directly regulates microneme secretion by activating PKA (Dawn et al. 2014).

A recent study describes a different mechanism for elevation of cytosolic $\left[\mathrm{Ca}^{2+}\right]$ in merozoites leading to microneme release. This study suggests the interaction of rhoptry protein PfRh1 to host RBCs triggers a surge in cytosolic $\left[\mathrm{Ca}^{2+}\right]$ levels in merozoites leading to release of microneme proteins. However, this model does not explain what triggers the release of PfRh1 from the rhoptries to the merozoite surface in the first place and implies that rhoptry secretion may precede microneme secretion (Gao et al. 2013).

The identification of some of the key players involved in signaling pathways leading to microneme and rhoptry discharge during invasion provides novel targets for intervention. For example, targeting PfCDPK1 with small molecule inhibitors will block release of key invasion-related microneme proteins to inhibit blood-stage parasite growth (Bansal et al. 2013). Given that enzymes such as PfCDPK1, which contain both the calmodulin-like $\mathrm{Ca}^{2+}$-binding domain and kinase domain, are of plant origin and are not found in mammalian cells suggests that it should be possible to design specific inhibitors 
Molecular Signaling by Malaria Parasites in the Bloodstream
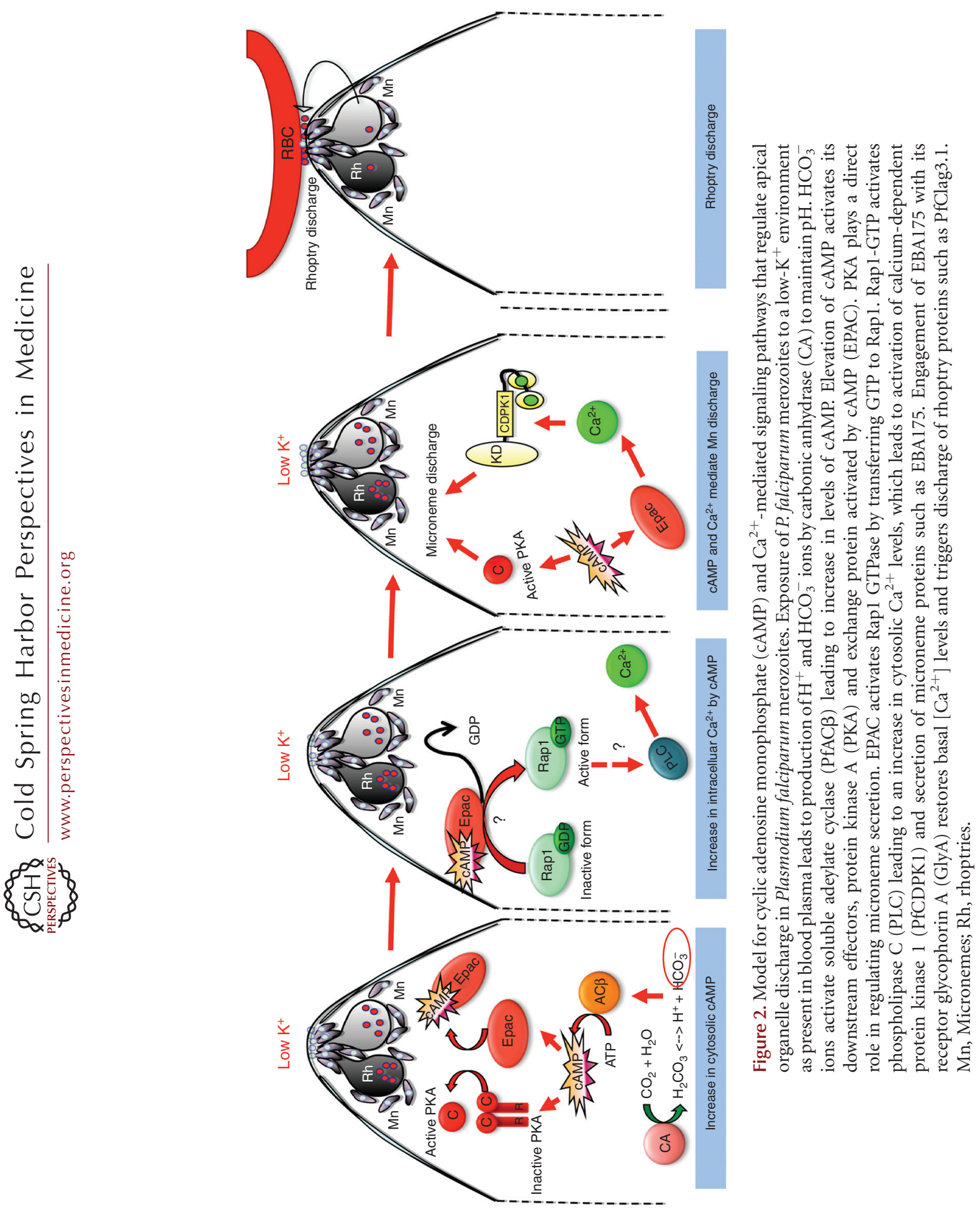
that block activation of PfCDPK1 following binding to $\mathrm{Ca}^{2+}$. Similarly other $P$. falciparum enzymes such as carbonic anhydrase (PfCA) or adenylate cyclase (PfAC $\beta$ ) could also be targeted to block production of second messengers required for transduction of signals that trigger microneme release to block blood-stage growth. The signaling pathways that lead to release of rhoptry proteins remain to be defined. Targeting both signaling pathways that are involved in microneme and rhoptry release may provide synergy to efficiently block erythrocyte invasion by apicomplexan pathogens.

\section{FUTURE PERSPECTIVES}

Significant progress has been made toward understanding the molecular basis of key steps in the biology of blood-stage malaria parasites such as RBC invasion and merozoite egress following replication in schizonts. As we identify the key parasite ligands involved in invasion, it is clear that it is unlikely that a single merozoite antigen will hold the key to the invasion process. Instead, we have learned that the invasion process is complex and has multiple steps with redundancies built into each step. So, there will be no key parasite ligand that can be targeted to block invasion. It is more likely that we will find combinations of antigens that are critical for invasion and yield synergy to block invasion with high efficiency when targeted together with a combination of specific antibodies. Such combinations may yield high rates of growth inhibition across diverse parasite strains and may provide the basis of highly effective blood-stage vaccines. Such vaccines may have an impact not only on blood-stage parasitemia but also on gametocyte densities leading to a reduction in transmission potential. Such vaccines that interrupt malaria transmission (VIMT) may be useful in elimination strategies in endemic regions in addition to providing protection against malaria.

The regulated secretion of key effector molecules from merozoite apical organelles plays an important role in the complex processes of host cell invasion and egress. A clear understanding of the complete signaling cascades that regulate apical organelle secretion is likely to reveal targets for small molecule inhibitors that can block invasion or egress. Such targets may provide attractive points for intervention to block blood-stage parasite growth. Some of the signaling pathways may be common to different stages. For example, development of gametes from gametocytes is also regulated by calcium and requires secretion of effectors such as perforin-like protein PfPLP2 for gamete egress. The signaling pathways that regulate processes such as gametocyte activation and gamete egress are not understood. If the signaling pathways that regulate steps such as vesicular secretion leading to egress of sexual-stage parasites are similar to those in blood stages, it may be possible to target both blood-stage growth and malaria transmission. Such interventions can not only protect against malaria but also assist in elimination of malaria by reducing malaria transmission.

\section{CONCLUDING REMARKS}

During blood-stage infection Plasmodium spp. merozoites repeatedly invade, multiply, exit, and reinvade host RBCs. The key steps of exit from mature schizonts and reinvasion of RBCs by merozoites requires timely regulation of key cellular processes such as apical organelle discharge, which is regulated by cAMP and $\mathrm{Ca}^{2+}$. dependent signal transduction pathways. The internal and external signals that regulate levels of second messengers such as cAMP and $\mathrm{Ca}^{2+}$ have been identified and some of the key players of the signaling pathways are known. A complete understanding of the signaling pathways that regulate the process of apical organelle secretion will enable the design of novel intervention strategies that block parasite egress or invasion to block blood-stage parasite growth. Some of the signaling pathways that regulate processes such as vesicular discharge in the blood stage may be common to other stages as well. It may thus be possible to target pathways that are common to both the blood and sexual stages to provide protection against malaria as well as block malaria transmission to eventually eliminate malaria. 


\section{ACKNOWLEDGMENTS}

We apologize to researchers whose work could not be cited because of space constraints. Work on erythrocyte invasion in our laboratories has been supported by the Department of Biotechnology (DBT), Government of India and European Commission (MalSig and EVIMalaR grants). S.S. is a recipient of the Innovative Young Biotechnology Award Grant from DBT.

\section{REFERENCES}

Abkarian M, Massiera G, Berry L, Roques M, BraunBreton C. 2011. A novel mechanism for egress of malarial parasites from red blood cells. Blood 117: $4118-4124$.

Agarwal S, Singh MK, Garg S, Chitnis CE, Singh S. 2012. $\mathrm{Ca}^{2+}$-mediated exocytosis of subtilisin-like protease 1: A key step in egress of Plasmodium falciparum merozoites. Cell Microbiol 15: 910-921.

Aikawa M, Miller LH, Johnson J, Rabbege J. 1978. Erythrocyte entry by malarial parasites. A moving junction between erythrocyte and parasite. J Cell Biol 77: 72-82.

Alam MM, Solyakov L, Bottrill AR, Flueck C, Siddiqui FA, Singh S, Mistry S, Viskaduraki M, Lee K, Hopp CS, et al. 2015. Phosphoproteomics reveals malaria parasite Protein Kinase $\mathrm{G}$ as a signalling hub regulating egress and invasion. 6: 7285.

Alleva LM, Kirk K. 2001. Calcium regulation in the intraerythrocytic malaria parasite Plasmodium falciparum. Mol Biochem Parasitol 117: 121-128.

Arastu-Kapur S, Ponder EL, Fonović UP, Yeoh S, Yuan F, Fonović M, Grainger M, Phillips CI, Powers JC, Bogyo M. 2008. Identification of proteases that regulate erythrocyte rupture by the malaria parasite Plasmodium falciparum. Nature Chem Biol 4: 203-213.

Bansal A, Singh S, More KR, Hans D, Nangalia K, Yogavel M, Sharma A, Chitnis CE. 2013. Characterization of Plasmodium falciparum calcium-dependent protein kinase 1 (PfCDPK1) and its role in microneme secretion during erythrocyte invasion. J Biol Chem 18: 1590-1602.

Bargieri D, Lagal V, Andenmatten N, Tardieux I, Meissner M, Ménard R. 2014. Host cell invasion by apicomplexan parasites: The junction conundrum. PLoS Pathog 10: e1004273.

Bartholdson SJ, Bustamante LY, Crosnier C, Johnson S, Lea S, Rayner JC, Wright GJ. 2012. Semaphorin-7A is an erythrocyte receptor for $P$. falciparum merozoite-specific TRAP homolog MTRAP. PLoS Pathog 8: e1003031.

Baum J. 2013. A complete molecular understanding of malaria parasite invasion of the human erythrocyte: Are we there yet? Pathog Glob Health 107: 107-110.

Besteiro S, Dubremetz JF, Lebrun M. 2011. The moving junction of apicomplexan parasites: A key structure for invasion. Cell Microbiol 13: 797-805.

Blackman MJ. 2004. Proteases in host cell invasion by the malaria parasite. Cell Microbiol 6: 893-903.
Blackman MJ. 2008. Malarial proteases and host cell egress: An "emerging" cascade. Cell Microbiol 10: 1925-1934.

Carruthers VB, Sibley LD. 1999. Mobilization of intracellularcalcium stimulates microneme discharge in Toxoplasma gondii. Mol Microbiol 31: 421-428.

Carruthers VB, Moreno SNJ, Sibley LD. 1999. Ethanol and acetaldehyde elevate intracellular $\left[\mathrm{Ca}^{2+}\right]$ and stimulate microneme discharge in Toxoplasma gondii. Biochem J 342: 379-386.

Cavasini CE, Mattos LC, Couto AA, Bonini-Domingos CR, Valencia SH, Neiras WC, Alves RT, Rossit AR, Castilho L, Machado RL. 2007. Plasmodium vivax infection among Duffy antigen-negative individuals from the Brazilian Amazon region: An exception? Trans $R$ Soc Trop Med Hyg 101: 1042-1044.

Chandramohanadas R, Davis PH, Beiting DP, Harbut MB, Darling C, Velmourougane G, Lee MY, Greer PA, Roos DS, Greenbaum DC. 2009. Apicomplexan parasites coopt host calpains to facilitate their escape from infected cells. Science 314: 794-797.

Chini EN, Nagamune K, Wetzel DM, Sibley LD. 2005. Evidence that the CADPR signalling pathway controls calcium-mediated microneme secretion in Toxoplasma gondii. Biochem J 389: 269-277.

Collins CR, Hackett F, Strath M, Penzo M, Withers-Martinez C, Baker DA, Blackman MJ. 2013. Malaria parasite cGMP-dependent protein kinase regulates blood stage merozoite secretory organelle discharge and egress. PLoS Pathog 9: e1003344.

Cowman AF, Crabb BS. 2006. Invasion of red blood cells by malaria parasites. Cell 124: 755-766.

Cowman AF, Berry D, Baum J. 2012. The cellular and molecular basis for malaria parasite invasion of the human red blood cell. J Cell Biol 198: 961-971.

Crosnier C, Bustamante LY, Bartholdson SJ, Bei AK, Theron M, Uchikawa M, Mboup S, Ndir O, Kwiatkowski DP, Duraisingh MT, et al. 2011. Basigin is a receptor essential for erythrocyte invasion by Plasmodium falciparum. $\mathrm{Na}$ ture 480: 534-537.

Dawn A, Singh S, More KR, Siddiqui FA, Pachikara N, Ramdani G, Langsley G, Chitnis CE. 2014. The central role of cAMP in regulating Plasmodium falciparum merozoite invasion of human erythrocytes. PLoS Pathog 10: e1004520.

Dvorak JA, Miller LH, Whitehouse WC, Shiroishi T. 1975. Invasion of erythrocytes by malaria merozoites. Science 187: $748-750$.

Dvorin JD, Martyn DC, Patel SD, Grimley JS, Collins CR, Hopp CS, Bright AT, Westenberger S, Winzeler E, Blackman MJ, et al. 2010. A plant-like kinase in Plasmodium falciparum regulates parasite egress from erythrocytes. Science 328: 910-912.

Fairlie WD, Spurck TP, McCoubrie JE, Gilson PR, Miller SK, McFadden GI, Malby R, Crabb BS, Hodder AN. 2008. Inhibition of malaria parasite development by a cyclic peptide that targets the vital parasite protein SERA5. Infect Immun 76: 4332-4344.

Farrell A, Thirugnanam S, Lorestani A, Dvorin JD, Eidell KP. 2012. A DOC2 protein identified by mutational profiling is essential for apicomplexan parasite exocytosis. Science 335: $218-221$. 
Gao X, Gunalan K, Yap SS, Preiser PR. 2013. Triggers of key calcium signals during erythrocyte invasion by Plasmodium falciparum. Nat Commun 4: 2862.

Garcia CR. 1999. $\mathrm{Ca}^{2+}$ homeostasis and signaling in the blood-stage malaria parasite. Parasitol Today 15: 488491.

Garg S, Agarwal S, Kumar S, Shams Yazdani S, Chitnis CE, Singh S. 2013. Calcium-dependent permeabilization of erythrocytes by a perforin-like protein during egress of malaria parasites. Nat Commun 4: 1736.

Gaur D, Chitnis CE. 2011. Molecular interactions and signaling mechanisms during erythrocyte invasion by malaria parasites. Curr Opin Microbiol 14: 422-428.

Gilson PR, Crabb BS. 2009. Morphology and kinetics of the three distinct phases of red blood cell invasion by Plasmodium falciparum merozoites. Int J Parasitol 39: 91-96.

Gloerich M, Bos JL. 2010. Epac: Defining a new mechanism for cAMP action. Annu Rev Pharmacol Toxicol 50: 355375.

Glushakova S, Mazar J, Hohmann-Marriott MF, Hama E, Zimmerberg J. 2008. Irreversible effect of cysteine protease inhibitors on the release of malaria parasites from infected erythrocytes. Cell Microbiol 11: 95-105.

Green JL, Rees-Channer RR, Howell SA, Martin SR, Knuepfer E, Taylor HM, Grainger M, Holder AA. 2008. The motor complex of Plasmodium falciparum: Phosphorylation by a $\mathrm{Ca}^{2+}$ dependent protein kinase. J Biol Chem 283: 30980-30989.

Hanssen E, Dekiwadia C, Riglar DT, Rug M, Lemgruber L, Cowman AF, Cyrklaff M, Kudryashev M, Frischknecht F, Baum J, et al. 2013. Electron tomography of Plasmodium falciparum merozoites reveals core cellular events that underpin erythrocyte invasion. Cell Microbiol 15: 1457-1472.

Hayton K, Gaur D, Liu A, Takahashi J, Henschen B, Singh S, Lambert L, Furuya T, Bouttenot R, Doll M. 2008. Erythrocyte binding protein PfRH5 polymorphisms determine species-specific pathways of Plasmodium falciparum invasion. Cell Host Microbe 4: 40-51.

Horuk R, Chitnis CE, Darbonne WC, Colby TJ, Rybicki A, Hadley TJ, Miller LH. 1993. A receptor for the malarial parasite Plasmodium vivax: The erythrocyte chemokine receptor. Science 261: 1182-1184.

Kafsack BF, Carruthers VB. 2010. Apicomplexan perforinlike proteins. Commun Integr Biol 3: 18-23.

Kafsack BF, Pena JD, Coppens I, Ravindran S, Boothroyd JC, Carruthers VB. 2009. Rapid membrane disruption by a perforin-like protein facilitates parasite exit from host cells. Science 323: 530-533.

Kato N, Sakata T, Breton G, Roch KGL, Nagle A, Andersen C, Bursulaya B, Henson K, Johnson J, Kumar KA, et al. 2008b. Gene expression signatures and small-molecule compounds link a protein kinase to Plasmodium falciparum motility. Nat Chem Biol 4: 347-356.

Kessler H, Herm-Götz A, Hegge S, Rauch M, Soldati-Favre D, Frischknecht F, Meissner M. 2008. Microneme protein 8-A new essential invasion factor in Toxoplasma gondii. J Cell Sci 121: 947e956.

Lavine MD, Arrizabalaga G. 2008. Exit from host cells by the pathogenic parasite Toxoplasma gondii does not require motility. Eukaryot Cell 7: 131-140.
Lourido S, Shuman J, Zhang C, Shokat KM, Hui R, Sibley LD. 2010. Calcium-dependent protein kinase 1 is an essential regulator of exocytosis in Toxoplasma. Nature 465: 359-362.

Lourido S, Tang K, Sibley LD. 2012. Distinct signalling pathways control Toxoplasma egress and host-cell invasion. EMBO J 31: 4524-4534.

Lovett JL, Marchesini N, Moreno SNJ, Sibley LD. 2002. Toxoplasma gondii microneme secretion involves intracellular $\mathrm{Ca}^{2+}$ release from IP3/ryanodine sensitive stores. J Biol Chem 277: 25870-25876.

McCoy JM, Whitehead L, van Dooren GG, Tonkin CJ. 2012. TgCDPK3 regulates calcium-dependent egress of Toxoplasma gondii from host cells. PLoS Pathog 8: e1003066.

Ménard D, Barnadas C, Bouchier C, Henry-Halldin C, Gray LR, Ratsimbasoa A, Thonier V, Carod JF, Domarle O, Colin Y. 2010. Plasmodium vivax clinical malaria is commonly observed in Duffy-negative Malagasy people. Proc Natl Acad Sci 107: 5967-5971.

Miller LH, Mason SJ, Clyde DF, McGinniss MH. 1976. The resistance factor to Plasmodium vivax in blacks. The Duffy-blood-group genotype, FyFy. N Engl J Med 295: $302-$ 304.

Millholland MG, Chandramohanadas R, Pizzarro A, Wehr A, Shi H, Darling C, Lim CT, Greenbaum DC. 2011. The malaria parasite progressively dismantles the host erythrocyte cytoskeleton for efficient egress. Mol Cell Proteomics 10: M111.010678.

Moon RW, Taylor CJ, Bex C, Schepers R, Goulding D, Janse CJ, Waters AP, Baker DA, Billker O. 2009. A cyclic GMP signalling module that regulates gliding motility in a malaria parasite. PLoS Pathog 5: e1000599.

Moreno SN, Docampo R. 2003. Calcium regulation in protozoan parasites. Curr Opin Microbiol 6: 359-364.

Moudy R, Manning TJ, Beckers CJ. 2001. The loss of cytoplasmic potassium upon host cell breakdown triggers egress of Toxoplasma gondii. J Biol Chem 276: 4149241501.

Nagamune K, Hicks LM, Fux B, Brossier F, Chini EN, Sibley LD. 2008. Abscisic acid controls calcium-dependent egress and development in Toxoplasma gondii. Nature 451: 207-210.

Olaya P, Wasserman M. 1991. Effect of calpain inhibitors on the invasion of human erythrocytes by the parasite Plasmodium falciparum. Biochim Biophys Acta 1096: 217221.

Richard D, MacRaild CA, Riglar DT, Chan JA, Foley M, Baum J, Ralph SA, Norton RS, Cowman AF. 2010. Interaction between Plasmodium falciparum apical membrane antigen 1 and the rhoptry neck protein complex defines a key step in the erythrocyte invasion process of malaria parasites. J Biol Chem 285: 14815-14822.

Riglar DT, Richard D, Wilson DW, Boyle MJ, Dekiwadia C, Turnbull L, Angrisano F, Marapana DS, Rogers KL, Whitchurch CB, et al. 2011. Super-resolution dissection of coordinated events during malaria parasite invasion of the human erythrocyte. Cell Host Microbe 9: 9-20.

Roiko MS, Carruthers VB. 2009. New roles for perforins and proteases in apicomplexan egress. Cell Microbiol 11: $1444-14452$. 
Roiko MS, Carruthers VB. 2013. Functional dissection of Toxoplasma gondii perforin-like protein 1 reveals a dual domain mode of membrane binding for cytolysis and parasite egress. J Biol Chem 288: 8712-8725.

Ryan JR, Stoute JA, Amon J, Dunton RF, Mtalib R, Koros J, Owour B, Luckhart S, Wirtz RA, Barnwell JW. 2006. Evidence for transmission of Plasmodium vivax among a Duffy antigen negative population in Western Kenya. Am J Trop Med Hyg 75: 575-581.

Salmon BL, Oksman A, Goldberg DE. 2001. Malaria parasite exit from the host erythrocyte: A two-step process requiring extraerythrocytic proteolysis. Proc Natl Acad Sci 98: 271-276.

Sharma P, Chitnis CE. 2013. Key molecular events during host cell invasion by Apicomplexan pathogens. Curr Opin Microbiol 16: 432-437.

Siddiqui FA, Dhawan S, Singh S, Singh B, Gupta P, Pandey A, Mohmmed A, Gaur D, Chitnis CE. 2013. A thrombospondin structural repeat containing rhoptry protein from Plasmodium falciparum mediates erythrocyte invasion. Cell Microbiol 15: 1341-1356.

Singh S, Chitnis CE. 2012. Signaling mechanism involved in apical organelle discharge during invasion of apicomplexan parasites. Microbes Infect 10: 820-824.

Singh S, Alam MM, Pal-Bhowmick I, Brzostowski JA, Chitnis CE. 2010. Distinct external signals trigger sequential release of apical organelles during erythrocyte invasion by malaria parasites. PLoS Pathog 6: e1000746.

Soni S, Dhawan S, Rosen KM, Chafel M, Chishti AH, Hanspal M. 2005. Characterization of events preceding the release of malaria parasite from the host red blood cell. Blood Cells Mol Dis 35: 201-211.

Srinivasan P, Beatty WL, Diouf A, Herrera R, Ambroggio X, Moch JK, Tyler JS, Narum DL, Pierce SK, Boothroyd JC, et al. 2011. Binding of Plasmodium merozoite proteins RON2 and AMA1 triggers commitment to invasion. Proc Natl Acad Sci 108: 13275-13280.

Taylor HM, McRobert L, Grainger M, Sicard A, Dluzewski AR, Hopp CS, Holder AA, Baker DA. 2010. The malaria parasite cyclic GMP-dependent protein kinase plays a central role in blood-stage schizogony. Eukaryot Cell 9: 37-45.

Tham WH, Wilson DW, Lopaticki S, Schmidt CQ, TettehQuarcoo PB, Barlow PN, Richard D, Corbin JE, Beeson JG, Cowman AF. 2010. Complement receptor 1 is the host erythrocyte receptor for Plasmodium falciparum PfRh4 invasion ligand. Proc Natl Acad Sci 107: 17327-17332.

Vaid A, Thomas DC, Sharma P. 2008. Role of $\mathrm{Ca}^{2+} / \mathrm{CaM}$ PfPKB signaling pathway in erythrocyte invasion by Plasmodium falciparum. J Biol Chem 283: 5589-5597.

Wickham ME, Culvenor JG, Cowman AF. 2003. Selective inhibition of a two step egress of malaria parasites from the host erythrocyte. J Biol Chem 278: 37658-37663.

Wiersma HI, Galuska SE, Tomley FM, Sibley LD, Liberator PA, Donald RG. 2004. A role for coccidian cGMP-dependent protein kinase in motility and invasion. Int J Parasitol 34: 369-380.

Winograd E, Clavijo CA, Bustamante LY, Jaramillo M. 1999. Release of merozoites from Plasmodium falciparum-infected erythrocytes could be mediated by a non-explosive event. Parasitol Res 85: 621-624.

Wu Y, Kuzma J, Maréchal E, Graeff R, Lee HC, Foster R, Chua NH. 1997. Abscisic acid signaling through cyclic ADP-ribose in plants. Science 278: 2126-2130.

Yeoh S, O’Donnell RA, Koussis K, Dluzewski AR, Ansell KH, et al. 2007. Subcellular discharge of a serine protease mediates release of invasive malaria parasites from host erythrocytes. Cell 131: 1072-1083. 


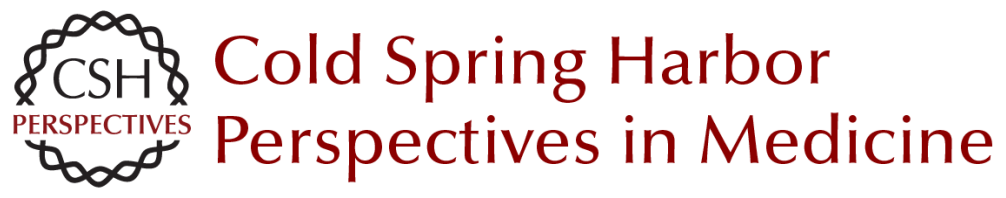

\title{
Molecular Signaling Involved in Entry and Exit of Malaria Parasites from Host Erythrocytes
}

\author{
Shailja Singh and Chetan E. Chitnis
}

Cold Spring Harb Perspect Med 2017; doi: 10.1101/cshperspect.a026815 originally published online May 15,2017

\section{Subject Collection Malaria: Biology in the Era of Eradication}

Modern Vector Control

Neil F. Lobo, Nicole L. Achee, John Greico, et al.

Anopheline Reproductive Biology: Impacts on Vectorial Capacity and Potential Avenues for Malaria Control

Sara N. Mitchell and Flaminia Catteruccia

Current and Future Prospects for Preventing

Malaria Transmission via the Use of Insecticides Hilary Ranson

Molecular Signaling Involved in Entry and Exit of Malaria Parasites from Host Erythrocytes Shailja Singh and Chetan E. Chitnis

Vaccines to Accelerate Malaria Elimination and Eventual Eradication

Julie Healer, Alan F. Cowman, David C. Kaslow, et al.

Immune Responses in Malaria Carole A. Long and Fidel Zavala

Antimalarial Drug Resistance: A Threat to Malaria Elimination

Didier Menard and Arjen Dondorp

Malaria during Pregnancy Michal Fried and Patrick E. Duffy
Malaria Pathogenesis

Danny A. Milner, Jr.

Determinants of Malaria Transmission at the

Population Level

Teun Bousema and Chris Drakeley

Host Cell Tropism and Adaptation of Blood-Stage

Malaria Parasites: Challenges for Malaria

Elimination

Caeul Lim, Selasi Dankwa, Aditya S. Paul, et al.

Malaria Transmission and Prospects for Malaria

Eradication: The Role of the Environment Marcia C. Castro

The Biology of Plasmodium vivax John H. Adams and Ivo Mueller

Malaria Genomics in the Era of Eradication Daniel E. Neafsey and Sarah K. Volkman

Malaria Epigenetics

Alfred Cortés and Kirk W. Deitsch

Malaria Parasite Liver Infection and

Exoerythrocytic Biology

Ashley M. Vaughan and Stefan H.I. Kappe

For additional articles in this collection, see http://perspectivesinmedicine.cshlp.org/cgi/collection/ 